\title{
Simvastatin-Induced Pleuro-Pericardial Effusion and Megaloblastic Anemia
}

\author{
Ali A. Alzu'bi* \\ Internal Medicine Department, King Hussein Medical City, Royal Medical Services, Jordan
}

“Corresponding author: Ali A. Alzu'bi, Internal Medicine Department, King Hussein Medical City, Royal Medical Services, Jordan, E-mail: ali.alzubi1985@yahoo.com

Receiving date: July 27 2015; Accepted date: August 19 2015; Published date: August 202015

Copyright: ( 2015 Alzu'bi AA. This is an open-access article distributed under the terms of the Creative Commons Attribution License, which permits unrestricted use, distribution, and reproduction in any medium, provided the original author and source are credited.

\begin{abstract}
Statins competitively inhibit 3-hyroxy-3-methylglutaryl coenzyme A (HMG CoA) reductase, an enzyme involved in cholesterol synthesis, especially in the liver. Statins are considered for all patients with cardiovascular diseases such as coronary heart disease, peripheral vascular disease, non-haemorrhagic stroke, and transient ischemic attacks. Pleural and pericardial effusions may be caused by infectious diseases, malignancies and autoimmune disorders. However, unexplained effusions should raise the possibility of drugs as being the cause. Drug-induced anemia may be in the form of auto-immune or oxidative hemolysis, aplastic anemia, sideroblastic anemia or megaloblastic anemia. I report a case of lymphocytic pleural effusion, pericardial effusion and megaloblastic anemia in a patient known to have ischemic stroke, diabetes mellitus and hypertension. In the view of un-yielding clinical and laboratory evaluation, a drug-induced reaction was suspected. Because of the recent addition of simvastatin to the patient's regimen, the improvement of the patient's condition after discontinuation of simvastatin and recurrence of symptoms following it's re-administration, a conclusion was made that simvastatin may be the cause of the patient's clinical picture and should be further considered as a cause of unexplained pleural and pericardial effusions, and megaloblastic anemia.
\end{abstract}

Keywords: Statins; Simvastatin; Drug-induced pleural effusion; Drug-induced pericardial effusion; Drug-induced megaloblastic anemia

\section{Introduction}

Statins, the usual prescribed lipid-lowering drugs, inhibit the HMG CoA reductase and increase the expression of low-density lipoprotein (LDL) receptors in the liver, allowing a reduction in the LDL serum levels and provide a significant decrease in cardiovascular mortality $[1,2]$. The commonest side-effects are muscle pain, myopathy, rhabdomyolysis, and elevations of hepatic enzyme [3]. Lupus-like syndromes, hypersensitivity pneumonitis and pseudopolymyositis, have been, also, reported [4]. Moreover, lovastatin was reported to cause hemolytic anemia [5], and pravastatin sodium has been reported to cause bilateral lymphocytic pleural effusion without any lung or extra-thoracic abnormalities [6].

Drug-induced hematologic disorders can affect any cell line, including white blood cells (WBCs), red blood cells (RBCs), and platelets. Drugs can affect RBCs by causing a number of different anemias, including drug-induced immune or oxidative hemolytic anemia, sideroblastic anemia, aplastic anemia, red cell aplasia, or druginduced megaloblastic anemia $[7,8]$.

Drug-induced pleural reactions may occur in the absence of parenchymal lung disease and usually manifest as pleural effusions. However, pleural thickening with or without pleural effusion, pleuritic chest pain, and in even fewer cases, pneumothorax or hemothorax, may, also, occur $[9,10]$. The pathological mechanisms explaining these reactions are not clear, but hypersensitivity reactions, oxidative stress of mesothelial cells, dose-dependent toxic effect, fluid retention and induction of chemical inflammation have all been proposed [10]. Generally, drug-induced lupus pleuritis is the most common cause of drug-induced pleural effusion and is associated with high erythrocyte sedimentation rate (ESR) and serum antinuclear antibodies (ANA) titers. Although histone antibodies are suggestive of the condition, with $50-80 \%$ occurrence, they are not specific [11].

\section{Case Report}

A 61 year old female patient with history of ischemic stroke, type 2 diabetes mellitus and hypertension, was admitted with pleuritic chest pain and progressive dyspnea for 4 weeks. She is non-smoker, and denies symptoms of cough, fever, myalgia, arthralgia, skin rashes, anorexia and weight loss. She had no history of allergy or exposure to agents that may harm the respiratory system. Her own medications, over the last 10 years, were metformin $(850 \mathrm{mg}$ TD), glibneclamide (5 $\mathrm{mg} \mathrm{TD})$, amlodipine (5 $\mathrm{mg} \mathrm{OD})$, enalapril (10 mg TD) and aspirin (100 mg OD). Simvastatin (20 mg OD) was added to her regimen, 3 months prior to her presenting symptoms, after she suffered an ischemic stroke.

Clinical examination was significant only for pallor, and decreased air entry bilaterally consistent with bilateral pleural effusion on chest $\mathrm{X}$-ray (Figure 1). On further evaluation, complete blood count (CBC) revealed anemia (hematocrit (Hct) level of 25\%, hemoglobin (HGB) level of $8.2 \mathrm{~g} / \mathrm{dl}$ and mean corpuscular volume (MCV) of $106 \mathrm{fL}$ ), normal WBCs $\left(6.650 / \mathrm{mm}^{3}\right)$ and normal platelets $(234,000 / \mathrm{mcL})$. There was no evidence of eosinophilia. ESR was elevated $(65 \mathrm{ml} / 1 \mathrm{st}$ hour). Chest CT scan was reported with moderate bilateral pleural effusion and pericardial effusion with normal lungs fields and no evidence of pulmonary embolism. 2D-Echocardiography revealed pericardial effusion of $2.3 \mathrm{~cm}$ posteriorly and $1.6 \mathrm{~cm}$ anteriorly, with no signs of ventricular compression, and $65 \%$ ejection fraction. Her blood film was significant for macrocytic RBCs, few hypersegmented neutrophiles and normal platelets.

Therapeutic and diagnostic pleurocentesis was done. Exudative effusion was evident with lactate dehydrogenase (LDH) level of 190 IU 
Page 2 of 3

(0.9 ratio) and total protein level of $3.9 \mathrm{~g}$ (0.56 ratio). Cellular and differential count revealed lymphocytes $65 \%$, neutrophils $33 \%$ and eosinophils $2 \%$. There were no malignant cells on cytological study. Polymerase chain reaction (PCR) for mycobacterium tuberculosis DNA was negative.

Erythrocytic hyperplasia, with other megaloblastic changes, was evident on bone marrow biopsy and aspirate. Serum folate and B12 levels, thyroid function, creatine kinase, kidney function and liver function, were all within normal ranges. Electrocardiography was normal with no changes suggestive of Pericarditis. Rheumatoid factor, ANA and anti-dsDNA, and $c$ and $p$ antineutrophil cytoplasmic antibodies were negative. Angiotensin converting enzyme level was 23 microgram/l.

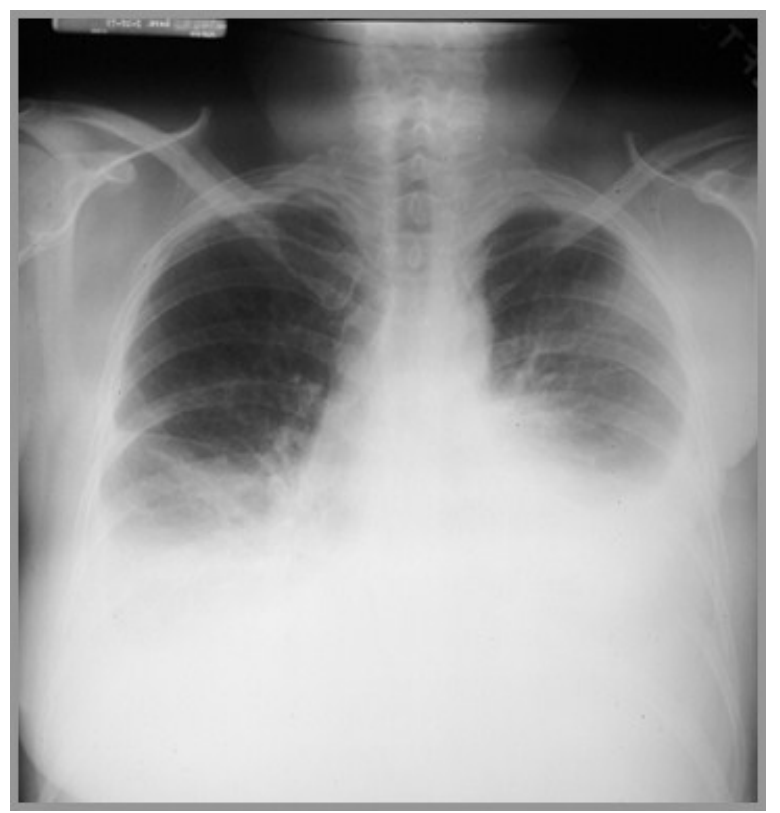

Figure 1: Chest X-ray showing bilateral pleural effusion.

In the view of her indeterminate clinical and laboratory findings, drug induced pathology was suspected. Because simvastatin was the most recent drug used in her regimen, it was discontinued. As the patient dyspnea was dramatically improved following pleurocentesis, and chest pain was improved following administration of paracetamol as needed; she was discharged home, with untouched pericardial effusion and without correction of anemia, and given appointment for follow up. 3 weeks later, the patient had no chest pain, even without pain killers, and had no dyspnea. Her Hct was 29\% and HGB was 9.7 $\mathrm{g} / \mathrm{dl}$. There were only obliterated costophrenic angles on chest X-ray with evident improvement of pericardial effusion $(1.9 \mathrm{~cm}$ posteriorly, $0.8 \mathrm{~cm}$ anteriorly). On next appointment, 1 month later; pleural effusion had disappeared, Hct was $33 \%$ and pericardial effusion was $0.7 \mathrm{~cm}$ posteriorly. 3 months later, the patient chest X-ray, $\mathrm{CBC}$ and 2$\mathrm{D}$ echocardiography were all normal and the patient was free of symptoms.

A re-challenge with simvastatin, for 3 weeks, had reproduce the symptoms of pleuritic chest pain and dyspnea and the patient developed pleural effusion, the drug was discontinued with dramatic improvement of symptoms over the next 4 weeks.

\section{Discussion}

Although drug induced pleural disease is generally underappreciated, its possibility should be emphasized in a patient with a pleural effusion that cannot be explained after the initial workup for common etiologies [11]. The usual presenting symptom in patients with drug-induced pleural disease is dyspnea, which may be due to a rapidly increasing pleural effusion or fibrosis, or may be due to an underlying parenchymal lung involvement occurring in association with pleural disease [10]. The onset of symptoms may be acute, soon after the exposure to a new drug, or may occur years later. However, a time gap of weeks to months is the usual [11]. A thorough and meticulous review of drug history, temporal relationship between exposure to a drug and symptoms occurrence, Disappearance of symptoms after discontinuation of a drug and reappearance after drug re-use and evidence of pleural fluid eosinophilia should raise the possibility of a drug-induced reaction [9,11]. Treatment of druginduced pleural disease is generally based on pleural fluid drainage to relieve dyspnea and on discontinuation of the offending drug, which is usually sufficient. Sometimes, when a hypersensitivity reaction is suspected or to reduce pleural inflammation, corticosteroids are prescribed. However, there are no sufficient data to prove corticosteroid efficiency [11].

Megaloblastic anemia, a condition that can be congenital or acquired, is associated with a hypercellular bone marrow with large, abnormal hematopoetic progenitor cells (megaloblasts). Pancytopenia may be present. Megaloblastic anemias are generally related to vitamin B12 or folic acid deficiencies resulting from malnutrition or defective absorption, however, megaloblastic anemias can, also, be druginduced [8]. Antimetabolites, alkylating agents and some antinucleosides used against HIV; which act by interfering with DNA synthesis, can all induce megaloblastic anemia [12]. Moreover, some drugs that inhibit dihydrofolate reductase, such as trimethoprim and pyrimethamine, have been associated with megaloblastic anemia primarily in patients at risk for folic acid deficiency. Sulfasalazine and anticonvulsants such as phenytoin interfere with absorption of folate and may induce megaloblastic anemia [8].

Simvastatin/ezetimibe was reported to cause lymphocytic pleural effusion with high ANA and without clinical signs of a rheumatologic syndrome [13]. Also, treatment with simvastatin had been reported to cause pleural effusions after 6 months to 13 yrs of therapy initiation $[14,15]$, but to the best of the present author's knowledge, this is the first case of pericardial effusion and megaloblastic anemia, with normal B12 and folate levels, expected to be caused by simvastatin. Furthermore, in the patient reported here, the absence of interstitial lung fibrosis, peripheral or pleural fluid eosinophilia, high ANA and abnormal liver function or weight loss, which were present in previously reported simvastatin-induced pleural effusions, suggests that different mechanisms could be responsible for pleural diseases induced by simvastatin.

\section{Conclusion}

Attributing a clinical condition to specific drug intake, after exclusion of other potential causes, may prove a complicated scenario. Statins intake should be considered as a cause of unexplained lymphocytic pleural effusion, pericardial effusion and megaloblastic anemia. In the view of normal serum folate and B12 levels, the mechanism of simvastatin-induced megaloblastic anemia needs further evaluation. 
Citation: Alzu'bi AA (2015) Simvastatin-Induced Pleuro-Pericardial Effusion and Megaloblastic Anemia. J Gen Practice 3: 198. doi: 10.4172/2329-9126.1000198

Page 3 of 3

\section{References}

1. Hebert PR, Gaziano JM, Chan KS, Hennekens CH (1997) Cholesterol lowering with statin drugs, risk of stroke, and total mortality: An overview of randomized trials. JAMA 278: 313-321.

2. Alberts AW (1988) Discovery, biochemistry and biology of lovastatin. Am J Cardiol 62: 10-15.

3. Liebhaber MI, Wright RS, Gelberg HJ, Dyer Z, Kupperman JL (1999) Polymyalgia, hypersensitivity pneumonitis and other reactions in patients receiving HMGCoA reductase inhibitors: A report of ten cases. Chest 115: 886-889.

4. Lantuejoul S, Brambilla E, Brambilla C, Devouassoux G (2002) Statininduced fibrotic nonspecific interstitial pneumonia. Eur Respir J 19: 577-580.

5. Robbins MJ, Iqbal A, Hershman R (1995) Lovastatin-induced hemolytic anemia: not a class-specific reaction. Am J Med 99: 328-329.

6. Kalomenidis I, Papiris S, Loukides S (2007) Bilateral pleural effusions associated with pravastatin sodium treatment. Eur Respir J 30: 1022.

7. Kamakshi V Rao (2014) Drug-Induced Hematologic Disorders, e/ Chapter 24, McGraw-Hill Education 359-374.
8. David M. Mintzer, Shira N. Billet, Chmielewski L (2009) Drug-Induced Hematologic Syndromes, Hindawi Publishing Corporation, Advances in Hematology, Volume.

9. Huggins JT, Sahn SA (2004) Drug-induced pleural disease, Clin Chest Med 25: 141-153.

10. Antony VB (1998) Drug-induced pleural disease. Clin Chest Med 19: 331-340.

11. Moschos C (2010) Ioannis KALOMENIDIS MD: drug related pleural disease, literature review: 8- 18.

12. Scott JM, Weir DG (1980) "Drug-induced megaloblastic change," Clinics in Haematology 9(3): 587-606.

13. Schwotzer R, Corti N, Ulrich S, Huber LC (2012) Simvastatin/ezetimibeinduced pleural effusion.

14. De Groot RE, Willems LN, Dijkman JH (1996) Interstitial lung disease with pleural effusion caused by simvastin. J Intern Med 239: 361-363.

15. Roncato-Sabéran M, Hustache-Mathieu L, Hoen B (2006) Eosinophilic pleural effusion caused by simvastatin after 13 years of exposure. Eur J Intern Med 17: 450. 